\title{
BINDING OF POLARONS AND ATOMS AT THRESHOLD
}

\author{
RUPERT L. FRANK, ELLIOTT H. LIEB, AND ROBERT SEIRINGER
}

\begin{abstract}
If the polaron coupling constant $\alpha$ is large enough, bipolarons or multi-polarons will form. When passing through the critical $\alpha_{c}$ from above, does the radius of the system simply get arbitrarily large or does it reach a maximum and then explodes? We prove that it is always the latter. We also prove the analogous statement for the Pekar-Tomasevich (PT) approximation to the energy, in which case there is a solution to the PT equation at $\alpha_{c}$. Similarly, we show that the same phenomenon occurs for atoms, e.g., helium, at the critical value of the nuclear charge. Our proofs rely only on energy estimates, not on a detailed analysis of the Schrödinger equation, and are very general. They use the fact that the Coulomb repulsion decays like $1 / r$, while 'uncertainty principle' localization energies decay more rapidly, as $1 / r^{2}$.
\end{abstract}

\section{INTRODUCTION}

We investigate the binding-unbinding transition of the ground state of multipolaron systems, both in the original Fröhlich model [6] and in the Pekar-Tomasevich large coupling approximation [18. The parameter that varies is the strength of the electron-electron Coulomb repulsion, conventionally denoted by $U>0$. (We could, alternatively, let the field coupling constant $\alpha$ vary, but it is mathematically convenient and equivalent to vary $U$.)

The conclusion of our rigorous analysis is that this transition is always 'firstorder' in accordance with a proposal of Verbist, Peeters and Devreese [22. That is, the Coulomb repulsion jumps discontinuously from a positive value to zero and the radius, too, jumps discontinuously.

The same thing happens for the helium atom unbinding as $U$ varies, as shown by M. and T. Hoffmann-Ostenhof and Simon [9]. Our analysis does not proceed, as in [9], by analyzing the Schrödinger equation, but, instead, is based on energy estimates. Indeed, our proof is also valid in the presence of a magnetic field; the proof in [9] cannot accommodate a magnetic field because it relies on the positivity of the ground state wave function. To illustrate our approach we first show how to reproduce the result of 9 as a warm-up to the harder polaron problem. The overall lesson is that this kind of discontinuous binding will occur whenever the net repulsion at large distances falls of slower than $r^{-2}$. This conclusion does not depend on Bose or Fermi statistics.

Date: June 2, 2011.

(C) 2011 by the authors. This paper may be reproduced, in its entirety, for non-commercial purposes. 
Fröhlich's Hamiltonian for $N$ particles interacting with the longitudinal distortions of a polar crystal is

$$
H_{U}^{(N)}=\sum_{i=1}^{N}\left(p_{i}^{2}-\sqrt{\alpha} \phi\left(x_{i}\right)\right)+H_{f}+U V_{C}(X)
$$

with $X=\left(x_{1}, \ldots, x_{N}\right) \in \mathbb{R}^{3 N}$ and

$$
V_{C}(X)=\sum_{i<j} \frac{1}{\left|x_{i}-x_{j}\right|} .
$$

The Hilbert space is $L^{2}\left(\mathbb{R}^{3 N}\right) \otimes \mathcal{F}$, where $\mathcal{F}$ is the bosonic Fock space for the longitudinal optical modes of the crystal, with scalar creation and annihilation operators $a^{\dagger}(k)$ and $a(k)$ satisfying $\left[a(k), a^{\dagger}\left(k^{\prime}\right)\right]=\delta\left(k-k^{\prime}\right)$. The phonon field energy is

$$
H_{f}=\int_{\mathbb{R}^{3}} d k a^{\dagger}(k) a(k)
$$

and the interaction of the crystal modes with the electron is

$$
\phi(x)=\frac{1}{\sqrt{2} \pi} \int_{\mathbb{R}^{3}} \frac{d k}{|k|}\left(e^{i k x} a(k)+e^{-i k x} a^{\dagger}(k)\right),
$$

with coupling constant $\alpha>0$. We define the ground state energy to be

$$
E_{U}^{(N)}(\alpha)=\inf \operatorname{spec} H_{U}^{(N)}=\inf _{\|\Psi\|=1}\left\langle\Psi\left|H_{U}^{(N)}\right| \Psi\right\rangle
$$

and the break-up energy to be

$$
\widetilde{E}_{U}^{(N)}(\alpha)=\min _{1 \leq n \leq N-1}\left(E_{U}^{(n)}(\alpha)+E_{U}^{(N-n)}(\alpha)\right) .
$$

It is always the case that $E_{U}^{(N)}(\alpha) \leq \widetilde{E}_{U}^{(N)}(\alpha)$. If there is strict inequality, we say that the $N$-particle system is bound.

A note on particle statistics. As we said, the results in this paper do not depend on Bose statistics (restrictions to symmetric spatial functions) or Fermi statistics (restrictions to anti-symmetric functions of space and spin). The numerical values of the binding energies and the discontinuities, etc., will depend on statistics, but not the qualitative features proved here.

Let us start by recalling some previous results. Some of these results are only valid in the Pekar-Tomasevich (PT) approximation, which will be described later.

(1) Physically, $U>2 \alpha$, but the case $U \leq 2 \alpha$ has been considered in 8 . They show that for fermions the energy satisfies $-C_{1} N^{7 / 3} \leq E_{U}^{(N)}(\alpha) \leq$ $-C_{2} N^{7 / 3}$. The same arguments for bosons lead to $-C_{1}^{\prime} N^{3} \leq E_{U}^{(N)}(\alpha) \leq$ $-C_{2}^{\prime} N^{3}$. In [1, 2] the sharp asymptotic constants for bosons and fermions are identified in the PT approximation.

(2) The case $U=2 \alpha$ is special. In the context of the PT model 8 showed that the energy behaves linearly in $N$ for fermions and in [2] an $N^{7 / 5}$ behavior for bosons is proved.

(3) For $U>2 \alpha$ the thermodynamic $\operatorname{limit}, \lim _{N \rightarrow \infty} E_{U}^{(N)}(\alpha) / N$, exists [5]. More importantly, it was shown in [5] that there is a value $U^{*}(\alpha)$ such that $E_{U}^{(N)}(\alpha)=N E^{(1)}(\alpha)$ for all $U \geq U^{*}(\alpha)$ and all $N$. (For $N=1$, we drop the index $U$ in the notation $E_{U}^{(N)}(\alpha)$.) In other words, there is no binding of any kind for $U>U^{*}(\alpha)$. 
(4) There is, in fact, binding for some range of $\alpha, U$ and $N$ with $U>2 \alpha$. (If $N=2$ and $\alpha$ is large then binding occurs for $2 \alpha<U \lesssim 2.3 \alpha$ [21, 19, 23.)

For every fixed $N$ and $\alpha$ there will generally be certain critical values of $U$, which we generically denote by $U_{c}$, at which binding will either appear or disappear. That is,

$$
E_{U}^{(N)}(\alpha)=\widetilde{E}_{U_{c}}^{(N)}(\alpha)
$$

and

$$
E_{U}^{(N)}(\alpha)<\widetilde{E}_{U}^{(N)}(\alpha)
$$

for either all $U \in\left(U_{c}-\delta, U_{c}\right)$ or all $U \in\left(U_{c}, U_{c}+\delta\right)$, for some $\delta>0$. The usual case is that there is a unique $U_{c}$ such that binding occurs for $U<U_{c}$ and no binding occurs for $U>U_{c}$, but we do not know if this is true and we certainly do not know how to prove it. The problem is that the energies of the break-up components also depend on $U$, so there is no obvious monotonicity.

The question we address here is what happens at $U_{c}$, or as $U$ approaches $U_{c}$ from the binding side. Does the compound simply explode into two pieces like a supernova or does it get larger and larger like a red giant? Our answer is that it always explodes, that is, the maximal distance between the particles stays finite. For the bipolaron this was seen variationally in [22] and stated as a general mathematical question in [16].

One would like to say that this implies the existence of an eigenfunction when $U=U_{c}$, but the polaron problem presumably never has bound states because of translation invariance [7]. (No doubt there are bound states of total momentum equal to zero, but we will not explore this here.) The PT problem, on the other hand, does have honest energy minimizers in a one-sided neighborhood of $U_{c}$ [11, [10, and we use the result about the finite maximal distance to show, in a separate theorem, that the PT problem has a minimizer when $U=U_{c}$.

In any case, a first order phase transition occurs. Suppose there is a break-up into two complexes of $n$ and $N-n$ particles each. Suppose particle one goes into the first complex and particle two goes into the second. Then their mutual Coulomb repulsion is zero after the break-up, but it is uniformly (in $U$ ) bounded away from zero before the break-up.

While in a physical situation one is usually not at the critical value of the parameters, the results proved here can be useful for a general understanding of quantum mechanics and, perhaps, for numerical analysis close to the critical values.

\section{The HeLIUM PROBLEM}

As a warm-up, we consider the ground state of a two-electron atom, of which helium and the hydrogen ion are examples. The Hamiltonian

$$
H_{U}=\sum_{i=1}^{2}\left(p_{i}^{2}-\left|x_{i}\right|^{-1}\right)+\frac{U}{\left|x_{1}-x_{2}\right|}
$$

acts on $L^{2}\left(\mathbb{R}^{6}\right)$. The ground state energy $E_{U}$ of $H_{U}$ is monotone increasing with respect to $U$, and there is a critical $U_{c}>0$ such that for $U<U_{c}, E_{U}$ is lower than

$$
-\frac{1}{4}=\inf \operatorname{spec}\left(p^{2}-|x|^{-1}\right) \text {, }
$$


which is the energy of a hydrogen atom, and for $U \geq U_{c}$ they are equal. Our general method, which will later be applied to the polaron problem, is illustrated by the following theorem of M. and T. Hoffmann-Ostenhof and Simon 9.

Theorem 1 (Binding for helium [9]). $H_{U_{c}}$ has a ground state eigenfunction $\psi_{U_{c}}$ in $L^{2}\left(\mathbb{R}^{6}\right)$, i.e., $H_{U_{c}} \psi_{U_{c}}=-\frac{1}{4} \psi_{U_{c}}$.

Remark 1. The proof uses only two facts about the operators $p^{2}$ and $|x|^{-1}$ : First, $p^{2}$ is non-negative and, second, the localization errors for $p^{2}$ fall-off faster than $|x|^{-1}$. The proof would work for many other combinations having this essential relationship. For instance, as mentioned in the introduction, $p^{2}$ could be replaced by $(p+A(x))^{2}$ for a suitable magnetic vector potential $A(x)$.

Theorem 1 follows by standard weak convergence arguments from the following Proposition 1. The details of the arguments, showing that the $H^{1}\left(\mathbb{R}^{6}\right)$-weak limit of ground states $\psi_{U}$ for $U \rightarrow U_{c}$ is not zero, are worked out in Section 5 on the PT equation, which is more complicated, in fact. Once one knows that the weak limit is not zero it is easy to see that this weak limit has to be a ground state.

We know from [3] that $U_{c}>1$, and therefore for the proof of Theorem 1 the assumption $U \geq 1+\delta$ in the following proposition is not really a restriction. The main point is the uniformity as $U \rightarrow U_{c}$.

Proposition 1 (Upper bound on the helium radius). For any $\delta>0$, there is a constant $C_{\delta}>0$ such that for all $1+\delta \leq U<U_{c}$ and for all normalized ground states $\psi_{U}$ of $H_{U}$ one has

$$
\left\langle\left.\psi_{U}|| x\right|_{\infty} ^{-1} \mid \psi_{U}\right\rangle \geq C_{\delta}
$$

where $|x|_{\infty}=\max \left\{\left|x_{1}\right|,\left|x_{2}\right|\right\}$.

Proof. Our goal will be to prove the following operator inequality. (Actually, the analogous statement for expectation values in the ground state would suffice for our purposes.) There is a radius $\ell_{0}>0$ and constants $c, C>0$ such that for all larger radii $\ell \geq \ell_{0}$ and for all $U \geq 1+\delta$ one has

$$
H_{U}-E_{U} \geq-\frac{C}{\ell^{2}} \theta\left(\ell-|x|_{\infty}\right)+\left(-\frac{1}{4}-E_{U}+\frac{c}{|x|_{\infty}}\right) \theta\left(|x|_{\infty}-\ell\right) .
$$

Here, $\theta$ is the Heaviside function, i.e., $\theta(t)=1$ if $t>0$ and $\theta(t)=0$ if $t<0$.

Since $E_{U} \leq-\frac{1}{4}$, this inequality, evaluated in a ground state $\psi_{U}$, implies

$$
\frac{C}{\ell^{2}} \int_{\left\{|x|_{\infty}<\ell\right\}} \psi_{U}^{2} d x \geq c \int_{\left\{|x|_{\infty}>\ell\right\}} \frac{\psi_{U}^{2}}{|x|_{\infty}} d x \quad \text { for all } \ell \geq \ell_{0} .
$$

Lemma 1 stated below, implies (2.1), and our proof is complete.

The crucial inequality (2.2) has a simple physical interpretation. The first term is a slightly negative energy, but vanishes relatively rapidly like $\ell^{-2}$. This leaves us with the second term, which is like a potential barrier. Although it goes to zero as $\ell \rightarrow \infty$, it does so much slower than $\ell^{-2}$ 'on average'. The significance of this is quantified by the following lemma.

Lemma 1 (Calculus lemma - simple version). Let $\rho \in L^{1}\left(\mathbb{R}_{+}\right)$be nonnegative, with $\int_{0}^{\infty} \rho(r) d r=1$. Assume that there are constants $b>0$ and $\ell_{c} \geq 0$ such that

$$
\frac{b}{\ell^{2}} \int_{0}^{\ell} \rho(r) d r \geq \int_{\ell}^{\infty} \frac{1}{r} \rho(r) d r
$$


for all $\ell \geq \ell_{c}$. Then

$$
\int_{0}^{\infty} \frac{1}{r} \rho(r) d r \geq \frac{1}{2\left(b+\ell_{c}\right)}
$$

and

$$
\int_{0}^{\ell_{c}} \rho(r) d r \geq \frac{\ell_{c}^{2}}{\left(b+\ell_{c}\right)^{2}}
$$

A generalization of this lemma is given later in Lemma 4

Proof of Lemma 1. We start by showing that

$$
\int_{\ell_{c}}^{\infty} \frac{1}{r} \rho(r) d r \geq \frac{1}{b+\ell_{c}}-\frac{1}{\ell_{c}} \int_{0}^{\ell_{c}} \rho(r) d r .
$$

To prove this, we define $f(R)=\int_{0}^{R} \rho(r) d r$ and rewrite the assumption as $b \ell^{-2} f(\ell) \geq$ $\int_{\ell}^{\infty} r^{-1} \rho(r) d r$ for all $\ell \geq \ell_{c}$. Therefore

$$
\begin{aligned}
\int_{\ell_{c}}^{\infty} \frac{1}{r} \rho(r) d r & =\int_{\ell_{c}}^{\infty} \frac{1}{r} f^{\prime}(r) d r=\int_{\ell_{c}}^{\infty} \frac{1}{r^{2}} f(r) d r-\frac{f\left(\ell_{c}\right)}{\ell_{c}} \\
& \geq b^{-1} \int_{\ell_{c}}^{\infty} \int_{r}^{\infty} \frac{1}{s} \rho(s) d s d r-\frac{f\left(\ell_{c}\right)}{\ell_{c}} \\
& =b^{-1} \int_{\ell_{c}}^{\infty} \frac{s-\ell_{c}}{s} \rho(s) d s-\frac{f\left(\ell_{c}\right)}{\ell_{c}} \\
& =b^{-1}\left(1-f\left(\ell_{c}\right)-\ell_{c} \int_{\ell_{c}}^{\infty} \frac{1}{s} \rho(s) d s\right)-\frac{f\left(\ell_{c}\right)}{\ell_{c}} .
\end{aligned}
$$

This is the same as (2.6).

In order to derive (2.4) from (2.6) we distinguish two cases according to whether $\ell_{c}^{-1} \int_{0}^{\ell_{c}} \rho(r) d r \leq\left(2\left(b+\ell_{c}\right)\right)^{-1}$ or not. In the first case, (2.6) yields

$$
\int_{0}^{\infty} \frac{1}{r} \rho(r) d r \geq \int_{\ell_{c}}^{\infty} \frac{1}{r} \rho(r) d r \geq \frac{1}{2\left(b+\ell_{c}\right)} .
$$

In the opposite case, we have

$$
\int_{0}^{\infty} \frac{1}{r} \rho(r) d r \geq \int_{0}^{\ell_{c}} \frac{1}{r} \rho(r) d r \geq \frac{1}{\ell_{c}} \int_{0}^{\ell_{c}} \rho(r) d r \geq \frac{1}{2\left(b+\ell_{c}\right)} .
$$

This proves (2.4).

Finally, to prove (2.5) we combine the assumption (2.3) at $\ell=\ell_{c}$ with (2.6) to get

$$
\frac{b}{\ell_{c}^{2}} \int_{0}^{\ell_{c}} \rho(r) d r \geq \int_{\ell_{c}}^{\infty} \frac{1}{r} \rho(r) d r \geq \frac{1}{b+\ell_{c}}-\frac{1}{\ell_{c}} \int_{0}^{\ell_{c}} \rho(r) d r
$$

which is the same as (2.5).

We now turn to the proof of the potential-theoretic inequality (2.2).

Lemma 2 (Partition of unity). For any $0<\epsilon<1 / 2$ there is a $C_{\epsilon}>0$ such that the following holds for any $\ell>0$. There is a quadratic partition of unity

$$
\chi_{0}(x)^{2}+\chi_{1}(x)^{2}+\chi_{2}(x)^{2}+\chi_{3}(x)^{2}=1 \quad \text { for all } x=\left(x_{1}, x_{2}\right) \in \mathbb{R}^{3} \times \mathbb{R}^{3},
$$


such that

$$
\begin{array}{ll}
\chi_{0}(x)=0 \text { unless } & |x|_{\infty} \leq \ell, \\
\chi_{1}(x)=0 \text { unless } & |x|_{\infty} \geq \ell / 2,|x|_{\infty} \leq(1-\epsilon)\left|x_{1}-x_{2}\right|, \\
\chi_{2}(x)=0 \text { unless } & |x|_{\infty} \geq \ell / 2,|x|_{\infty} \geq(1-2 \epsilon)\left|x_{1}-x_{2}\right|,\left|x_{1}\right| \leq(1+\epsilon)\left|x_{2}\right|, \\
\chi_{3}(x)=0 \text { unless } & |x|_{\infty} \geq \ell / 2,|x|_{\infty} \geq(1-2 \epsilon)\left|x_{1}-x_{2}\right|,\left|x_{2}\right| \leq(1+\epsilon)\left|x_{1}\right|,
\end{array}
$$

and

$$
\begin{aligned}
& \sum_{j}\left|\nabla \chi_{j}\right|^{2} \leq \frac{C_{\epsilon}}{\ell^{2}} \quad \text { when } \quad \chi_{0}(x)^{2}>0, \\
& \sum_{j}\left|\nabla \chi_{j}\right|^{2} \leq \frac{C_{\epsilon}}{\ell|x|_{\infty}} \quad \text { when } \quad \chi_{1}(x)^{2}+\chi_{2}(x)^{2}+\chi_{3}(x)^{2}>0 .
\end{aligned}
$$

One can choose $\chi_{0}$ and $\chi_{1}$ to be symmetric functions of $\left(x_{1}, x_{2}\right)$ and one can choose $\chi_{2}\left(x_{1}, x_{2}\right)=\chi_{3}\left(x_{2}, x_{1}\right)$. This preserves both Bose and Fermi statistics.

Sketch of proof of Lemma 专. The localization error $\sum_{j}\left|\nabla \chi_{j}\right|^{2}$ is supported in three regions, $\left\{\ell / 2 \leq|x|_{\infty} \leq \ell\right\},\left\{(1-2 \epsilon)\left|x_{1}-x_{2}\right| \leq|x|_{\infty} \leq(1-\epsilon)\left|x_{1}-x_{2}\right|\right\}$ and $\left\{(1+\epsilon)^{-1}\left|x_{2}\right| \leq\left|x_{1}\right| \leq(1+\epsilon)\left|x_{2}\right|\right\}$. It is a geometric question to check the stated inequalities on the supports of the different $\chi$ 's. We leave this to the reader. The fact that $C_{\epsilon}$ is independent of $\ell$ is a consequence of scaling.

Proof of inequality (2.2). By Lemma 2 and the IMS localization formula we can write, for any wave function $\Psi$,

$$
\left\langle\Psi\left|H_{U}\right| \Psi\right\rangle=\sum_{j=0}^{3}\left\langle\left.\Psi_{j}\left|H_{U}-\sum_{k=0}^{3}\right| \nabla \chi_{k}\right|^{2} \mid \Psi_{j}\right\rangle=: \sum_{j=0}^{3} e_{j}\left\|\Psi_{j}\right\|^{2}
$$

with $\Psi_{j}\left(x_{1}, x_{2}\right)=\Psi\left(x_{1}, x_{2}\right) \chi_{j}\left(x_{1}, x_{2}\right)$ and with numbers $e_{j}$ (depending on $\Psi_{j}$ ). In the following, we shall derive lower bounds on $e_{j}$.

For $j=0$, we simply use the bound on the localization error from Lemma 2 to bound

$$
e_{0} \geq E_{U}-\frac{C_{\epsilon}}{\ell^{2}}
$$

For $j=1$, we use the fact that on the support of $\chi_{1}$, if, say, $\left|x_{2}\right|=|x|_{\infty}$ then

$$
\left|x_{1}\right| \geq \frac{\epsilon}{1-\epsilon}\left|x_{2}\right|=\frac{\epsilon}{1-\epsilon}|x|_{\infty} .
$$

Therefore, for any $\lambda \geq 0$,

$$
-\left|x_{1}\right|^{-1}-\left|x_{2}\right|^{-1} \geq-\epsilon^{-1}|x|_{\infty}^{-1} \geq-2\left(\epsilon^{-1}+\lambda\right) \ell^{-1}+\lambda|x|_{\infty}^{-1}
$$

and

$$
e_{1} \geq-2\left(\epsilon^{-1}+\lambda\right) \ell^{-1}+\left\|\Psi_{1}\right\|^{-2}\left\langle\Psi_{1}\left|\frac{\lambda-C_{\epsilon} \ell^{-1}}{|x|_{\infty}}\right| \Psi_{1}\right\rangle .
$$

For $j=2$ we bound $p_{1}^{2}-\left|x_{1}\right|^{-1} \geq-\frac{1}{4}$. Moreover, in that region

$$
\left|x_{2}\right| \geq(1+\epsilon)^{-1}|x|_{\infty} \quad \text { and } \quad\left|x_{1}-x_{2}\right| \leq(1-2 \epsilon)^{-1}|x|_{\infty},
$$

so that

$$
e_{2} \geq-\frac{1}{4}+\left\|\Psi_{2}\right\|^{-2}\left\langle\Psi_{2}\left|\frac{U(1-2 \epsilon)-1-\epsilon-C_{\epsilon} \ell^{-1}}{|x|_{\infty}}\right| \Psi_{2}\right\rangle
$$


Similarly, for $j=3$,

$$
e_{3} \geq-\frac{1}{4}+\left\|\Psi_{3}\right\|^{-2}\left\langle\Psi_{3}\left|\frac{U(1-2 \epsilon)-1-\epsilon-C_{\epsilon} \ell^{-1}}{|x|_{\infty}}\right| \Psi_{3}\right\rangle .
$$

In order to specify the free parameters $\epsilon, \ell$ and $\lambda$ we restrict ourselves to $U \geq 1+\delta$ with some fixed $\delta>0$. (We will not reflect the dependence on $\delta$ in our notation.) First, we choose $\epsilon$ so small that $U(1-2 \epsilon)-1-\epsilon \geq \delta / 2$ and we choose $\lambda=\delta / 2$. Then we choose $\ell_{0}$ so large that $2\left(\epsilon^{-1}+\lambda\right) \ell_{0}^{-1} \leq 1 / 4$ and $C_{\epsilon} \ell_{0}^{-1} \leq \delta / 4$. With these choices, we have

$$
e_{j} \geq-\frac{1}{4}+\left\|\Psi_{j}\right\|^{-2}\left\langle\Psi_{j}\left|\frac{\delta / 4}{|x|_{\infty}}\right| \Psi_{j}\right\rangle
$$

for any $\ell \geq \ell_{0}$ and any $j=1,2,3$. This, together with the fact that

$$
\chi_{0}(x)^{2} \leq \theta\left(\ell-|x|_{\infty}\right)
$$

implies (2.2).

This completes our proof of Proposition 1.

Remark 2. One can ask a similar question for $N$ electrons and whether there is binding at the (unique) $U=U_{c}$ such that

$$
E_{U_{c}}^{(N)}=E_{U_{c}}^{(N-1)} .
$$

(Here $E_{U}^{(n)}$ is the ground state energy of $n$ electrons with repulsion strength $U$.) We denote by $M$ the smallest number $n \leq N-1$ such that $E_{U_{c}}^{(N)}=E_{U_{c}}^{(n)}$ and we recall that by Zhislin's theorem [24] we have $U_{c} \geq M^{-1}$. Whether equality or strict inequality holds has to be decided by an independent variational calculation (as Bethe [3] did for helium). We have nothing to say about the case $U_{c}=M^{-1}$. On the other hand, if $U_{c}>M^{-1}$ our method should work and show that there is a bound state for the $N$-electron system. Again, the method should extend to magnetic fields, pseudo-relativistic models with positive mass, spin-polarized systems, etc.

Another area in which to try to utilize our method is Hartree or Hartree-Fock theory, though we have not pursued this.

\section{The Bipolaron}

We now return to our main theme and consider the simplest interesting case, namely two polarons, whose Hamiltonian is

$$
H_{U}^{(2)}=p_{1}^{2}+p_{2}^{2}-\sqrt{\alpha} \phi\left(x_{1}\right)-\sqrt{\alpha} \phi\left(x_{2}\right)+H_{f}+\frac{U}{\left|x_{1}-x_{2}\right|} .
$$

We have shown in [5] that there is a critical $U_{c}(\alpha)<\infty$ such that $E_{U}^{(2)}(\alpha)=$ $2 E^{(1)}(\alpha)$ for all $U \geq U_{c}(\alpha)$, while $E_{U}^{(2)}(\alpha)<2 E^{(1)}(\alpha)$ for all $U<U_{c}(\alpha)$ (by concavity). It is easy to see that $U_{c}(\alpha) \geq 2 \alpha$. In the following, we assume that $U_{c}(\alpha)>2 \alpha$. This is true for large $\alpha$, since $\liminf _{\alpha \rightarrow \infty} U_{c}(\alpha) / \alpha \gtrsim 2.3$ due to the convergence to the Pekar-Tomasevich functional in the strong coupling limit [4, 14, 16, for which the critical ratio is known to be at least $\approx 2.3$ [21, 19, 23. 
Theorem 2 (Upper bound on the bipolaron radius). For any $\epsilon>0$, there is a constant $C_{\epsilon}>0$ such that for all $0<2 \alpha(1+\epsilon)<U<U_{c}(\alpha)$ and for all states $\Psi$ one has

$$
\left\langle\Psi\left|\frac{1}{\left|x_{1}-x_{2}\right|}\right| \Psi\right\rangle \geq \frac{U-2 \alpha(1+\epsilon)}{C_{\epsilon}(1+U / \alpha)} \frac{\left\langle\Psi\left|2 E^{(1)}(\alpha)-H_{U}^{(2)}\right| \Psi\right\rangle}{2 E^{(1)}(\alpha)-E_{U}^{(2)}(\alpha)} .
$$

We emphasize two consequences of Theorem 2

(1) In any approximate ground state, in the sense that

$$
\left\langle\Psi\left|H_{U}^{(2)}\right| \Psi\right\rangle \leq(1-\lambda) 2 E^{(1)}(\alpha)+\lambda E_{U}^{(2)}(\alpha)
$$

for some $\lambda>0$, the expectation value of $\left|x_{1}-x_{2}\right|^{-1}$ is uniformly bounded from below by a positive number as $U$ increases to $U_{c}(\alpha)$. In particular, the size of the bipolaron does not increase indefinitely as $U \rightarrow U_{c}(\alpha)$.

(2) The bipolaron energy $E_{U}^{(2)}(\alpha)$ is not differentiable in $U$ at $U=U_{c}(\alpha)$. While the right derivative is zero, the left derivative is at least as big as $\left(U_{c}(\alpha)-2 \alpha(1+\epsilon)\right) /\left[C_{\epsilon}\left(1+U_{c}(\alpha) / \alpha\right)\right]$, for any $\epsilon>0$.

Proof. The proof of Theorem 2 will be divided into three parts.

Step 1. Partition of the interparticle distance. As in [5] we choose a quadratic partition of unity and localize the particles according to their relative distance. In order to construct this partition, we pick some parameters $b>1$ and $\ell>0$, and let

$$
\varphi(t):= \begin{cases}0 & \text { for } t \leq \ell / b, \\ \sin \frac{\pi}{2} \frac{t-\ell / b}{\ell-\ell / b} & \text { for } \ell / b \leq t \leq \ell, \\ \cos \frac{\pi}{2} \frac{t-\ell}{b \ell-\ell} & \text { for } \ell \leq t \leq b \ell, \\ 0 & \text { for } t \geq b \ell .\end{cases}
$$

For $j \geq 1$, let $\varphi_{j}(t):=\varphi\left(b^{1-j} t\right)$, and for $j=0$, let

$$
\varphi_{0}(t):= \begin{cases}1 & \text { for } t \leq \ell / b \\ \cos \frac{\pi}{2} \frac{t-\ell / b}{\ell-\ell / b} & \text { for } \ell / b \leq t \leq \ell \\ 0 & \text { for } t \geq \ell\end{cases}
$$

Then

$$
\sum_{j \geq 0} \varphi_{j}(t)^{2}=1 \quad \text { for all } t \geq 0
$$

Using the IMS localization formula, we can write, for any wave function $\Psi$,

$$
\left\langle\Psi\left|H_{U}^{(2)}\right| \Psi\right\rangle=\sum_{j \geq 0}\left\langle\left.\Psi_{j}\left|H_{U}^{(2)}-2 \sum_{k \geq 0}\right| \varphi_{k}^{\prime}\left(\left|x_{1}-x_{2}\right|\right)\right|^{2} \mid \Psi_{j}\right\rangle=: \sum_{j \geq 0} e_{j}\left\|\Psi_{j}\right\|^{2}
$$

with $\Psi_{j}\left(x_{1}, x_{2}\right)=\Psi\left(x_{1}, x_{2}\right) \varphi_{j}\left(\left|x_{1}-x_{2}\right|\right)$ and with numbers $e_{j}$ (depending on $\Psi_{j}$ ). In the following, we shall derive lower bounds on $e_{j}$. For our bounds we shall use the fact that on the support of $\varphi_{j}\left(\left|x_{1}-x_{2}\right|\right)$, the localization error is dominated by

$$
\sum_{k \geq 0}\left|\varphi_{k}^{\prime}\left(\left|x_{1}-x_{2}\right|\right)\right|^{2} \leq \frac{\pi^{2}}{4(\ell-\ell / b)^{2}} \times \begin{cases}1 & \text { if } j=0 \\ b^{2(1-j)} & \text { if } j \geq 1\end{cases}
$$


Step 2. Estimate of localized energies. Because of (3.8) we can simply bound

$$
e_{0} \geq E_{U}^{(2)}(\alpha)-\frac{\pi^{2}}{2(\ell-\ell / b)^{2}}
$$

For $j \geq 1$, we further localize each of the two particles to its own ball of radius $b^{j} L$ for some parameter $L>0$. This will entail an additional localization error. Concretely, let

$$
\chi(x)=\frac{1}{\sqrt{2 \pi}|x|} \begin{cases}\sin (\pi|x|) & \text { for }|x| \leq 1 \\ 0 & \text { for }|x| \geq 1\end{cases}
$$

and note that $\int d x \chi(x)^{2}=1$ and $\int d x|\nabla \chi(x)|^{2}=\pi^{2}$. With

$$
\Psi_{j, u_{1}, u_{2}}\left(x_{1}, x_{2}\right)=\Psi_{j}\left(x_{1}, x_{2}\right)\left(b^{j} L\right)^{-3} \chi\left(b^{-j}\left(x_{1}-u_{1}\right) / L\right) \chi\left(b^{-j}\left(x_{2}-u_{2}\right) / L\right)
$$

we have, by a continuous version of the IMS localization formula,

$$
\left\langle\Psi_{j}\left|H_{U}^{(2)}\right| \Psi_{j}\right\rangle=\int_{\mathbb{R}^{3}} d u_{1} \int_{\mathbb{R}^{3}} d u_{2}\left\langle\Psi_{j, u_{1}, u_{2}}\left|H_{U}^{(2)}-\frac{2 \pi^{2}}{b^{2 j} L^{2}}\right| \Psi_{j, u_{1}, u_{2}}\right\rangle .
$$

Note that since $b^{j} \ell \geq\left|x_{1}-x_{2}\right| \geq b^{j-2} \ell$ on the support of $\varphi_{j}$, the wave function $\Psi_{j, u_{1}, u_{2}}$ is non-zero only if the distance $d$ between the two balls of radius $b^{j} L$ centered at $u_{1}$ and $u_{2}$, respectively, satisfies

$$
d \in D_{j}:=\left[b^{j-2} \ell-4 b^{j} L, \ell b^{j}\right] .
$$

We shall choose $L<\ell /\left(4 b^{2}\right)$.

Lemma 3. Assume that $\Psi$ is normalized and supported in $B_{1} \times B_{2}$ where $B_{1}$ and $B_{2}$ are disjoint balls of some radius $R$, separated a distance $d$. Then

$$
\left\langle\Psi\left|H_{U}^{(2)}\right| \Psi\right\rangle \geq 2 E^{(1)}(\alpha)-\frac{2 \alpha}{d}+\frac{U}{d+4 R} .
$$

Eq. (3.14) is an easy consequence of the functional integral representation of the ground state energy. The proof can be found in [5, Lemma 1].

We shall apply inequality (3.14) to (3.12), with $U$ replaced by $2 \alpha(1+\epsilon)$ for some $\epsilon>0$. Using also (3.8) we conclude that

$$
\begin{aligned}
e_{j} \geq & 2 E^{(1)}(\alpha)+\left\|\Psi_{j}\right\|^{-2}\left\langle\Psi_{j}\left|\frac{U-2 \alpha(1+\epsilon)}{\left|x_{1}-x_{2}\right|}\right| \Psi_{j}\right\rangle \\
& +\min _{d \in D_{j}}\left(-\frac{2 \alpha}{d}+\frac{2 \alpha(1+\epsilon)}{d+4 b^{j} L}\right)-b^{-2 j}\left(\frac{b^{2} \pi^{2}}{2(\ell-\ell / b)^{2}}+\frac{2 \pi^{2}}{L^{2}}\right) .
\end{aligned}
$$

For given $\epsilon>0$ (and given $b>1$ ), we can choose $L$ to be a small enough constant times $\ell$ such that, as long as $\ell$ is large enough, the sum of the terms in the second line of (3.15) is positive. More precisely, for given $\epsilon>0$ (and given $b>1$ ) we can choose $\delta>0$ small enough such that

$$
\kappa_{\epsilon}:=\min _{b^{-2}-4 \delta \leq d \leq 1}\left(-\frac{1}{d}+\frac{1+\epsilon}{d+4 \delta}\right)>0 .
$$

With $L=\delta \ell$, we see that

$$
\min _{d \in D_{j}}\left(-\frac{2 \alpha}{d}+\frac{2 \alpha(1+\epsilon)}{d+4 b^{j} L}\right)-b^{-2 j}\left(\frac{b^{2} \pi^{2}}{2(\ell-\ell / b)^{2}}+\frac{2 \pi^{2}}{L^{2}}\right) \geq 0
$$

for all $j \geq 1$ if

$$
\ell \geq \ell_{c}:=\frac{\pi^{2}}{\alpha \kappa_{\epsilon}}\left(\frac{b}{4\left(1-b^{-1}\right)^{2}}+\frac{1}{b \delta^{2}}\right)
$$


Under this condition, we thus have

$$
e_{j} \geq 2 E^{(1)}(\alpha)+\left\|\Psi_{j}\right\|^{-2}\left\langle\Psi_{j}\left|\frac{U-2 \alpha(1+\epsilon)}{\left|x_{1}-x_{2}\right|}\right| \Psi_{j}\right\rangle
$$

for all $j \geq 1$.

Step 3. Upper bound on the particle distance. From (3.7) and our bounds (3.9) and (3.19) on $e_{j}$ we conclude that, for all $\ell \geq \ell_{c}$,

$$
\begin{aligned}
H_{U}^{(2)}-2 E^{(1)}(\alpha) \geq & \left(E_{U}^{(2)}(\alpha)-2 E^{(1)}(\alpha)-\frac{\pi^{2}}{2 \ell^{2}\left(1-b^{-1}\right)^{2}}\right) \varphi_{0}\left(\left|x_{1}-x_{2}\right|\right)^{2} \\
& +\frac{U-2 \alpha(1+\epsilon)}{\left|x_{1}-x_{2}\right|}\left(1-\varphi_{0}\left(\left|x_{1}-x_{2}\right|\right)^{2}\right) \\
\geq & \left(E_{U}^{(2)}(\alpha)-2 E^{(1)}(\alpha)-\frac{\pi^{2}}{2 \ell^{2}\left(1-b^{-1}\right)^{2}}\right) \theta\left(\ell-\left|x_{1}-x_{2}\right|\right) \\
& +\frac{U-2 \alpha(1+\epsilon)}{\left|x_{1}-x_{2}\right|} \theta\left(\left|x_{1}-x_{2}\right|-\ell\right) .
\end{aligned}
$$

Now let $\Psi$ satisfy

$$
\left\langle\Psi\left|H_{U}^{(2)}-2 E^{(1)}(\alpha)\right| \Psi\right\rangle \leq \lambda\left(E_{U}^{(2)}(\alpha)-2 E^{(1)}(\alpha)\right)
$$

for some $0<\lambda<1$. For such a $\Psi$, the previous inequality yields the bound

$$
\begin{aligned}
& \left\langle\Psi\left|\left[(1-\lambda)\left(2 E^{(1)}(\alpha)-E_{U}^{(2)}(\alpha)\right)+\frac{\pi^{2}}{2 \ell^{2}\left(1-b^{-1}\right)^{2}}\right] \theta\left(\ell-\left|x_{1}-x_{2}\right|\right)\right| \Psi\right\rangle \\
& \geq\left\langle\Psi\left|\left[\lambda\left(2 E^{(1)}(\alpha)-E_{U}^{(2)}(\alpha)\right)+\frac{U-2 \alpha(1+\epsilon)}{\left|x_{1}-x_{2}\right|}\right] \theta\left(\left|x_{1}-x_{2}\right|-\ell\right)\right| \Psi\right\rangle .
\end{aligned}
$$

To conclude the proof, we need the following lemma.

Lemma 4 (Calculus lemma - general version). Let $\rho \in L^{1}\left(\mathbb{R}_{+}\right)$be nonnegative, with $\int_{0}^{\infty} \rho(r) d r=1$. Assume that there are constants $a \geq 0, b>0, c>0$, $0<\lambda \leq 1$ and $\ell_{c} \geq 0$ such that

$$
\left((1-\lambda) a+\frac{b}{\ell^{2}}\right) \int_{0}^{\ell} \rho(r) d r \geq \int_{\ell}^{\infty}\left(\lambda a+\frac{c}{r}\right) \rho(r) d r
$$

for all $\ell \geq \ell_{c}$. Then

$$
\int_{0}^{\infty} \frac{1}{r} \rho(r) d r \geq \frac{\lambda c}{b+2 c \ell_{c}}
$$

Proof. Let $f(r)=\int_{0}^{r} \rho(s) d s$, and define $0<\ell_{0} \leq \infty$ by $f\left(\ell_{0}\right)=\lambda$. In case $\ell_{0} \leq \ell_{c}$, we have $f\left(\ell_{c}\right) \geq \lambda$ and hence

$$
\int_{0}^{\infty} \frac{1}{r} \rho(r) d r \geq \frac{f\left(\ell_{c}\right)}{\ell_{c}} \geq \frac{\lambda}{\ell_{c}} .
$$

In particular, (3.24) holds. We can thus assume $\ell_{0}>\ell_{c}$. For all $\ell \leq \ell_{0}$, we have

$$
(1-\lambda) f(\ell) \leq \lambda(1-f(\ell))
$$

and hence (3.23) implies that

$$
\frac{b}{\ell^{2}} f(\ell) \geq c \int_{\ell}^{\infty} \frac{1}{r} \rho(r) d r
$$


for all $\ell_{c} \leq \ell \leq \ell_{0}$. Using integration by parts,

$$
\int_{0}^{\infty} \frac{1}{r} \rho(r) d r \geq \frac{f\left(\ell_{c}\right)}{\ell_{c}}+\int_{\ell_{c}}^{\ell_{0}} \frac{1}{r} f^{\prime}(r) d r=\frac{f\left(\ell_{0}\right)}{\ell_{0}}+\int_{\ell_{c}}^{\ell_{0}} \frac{1}{r^{2}} f(r) d r .
$$

The first term on the right can be dropped for a lower bound, and in the integrand of the second we can use (3.27). We obtain

$$
\int_{0}^{\infty} \frac{1}{r} \rho(r) d r \geq \frac{c}{b} \int_{\ell_{c}}^{\ell_{0}} d r \int_{r}^{\infty} \frac{f^{\prime}(s)}{s} d s=\frac{c}{b} \int_{\ell_{c}}^{\infty} \frac{f^{\prime}(s)}{s}\left(\min \left\{s, \ell_{0}\right\}-\ell_{c}\right) d s .
$$

For a lower bound, we can restrict the integral on the right to $s \leq \ell_{0}$. Using (3.25) again, this yields

$$
\begin{aligned}
\int_{0}^{\infty} \frac{1}{r} \rho(r) d r & \geq \frac{c}{b} \int_{\ell_{c}}^{\ell_{0}} \frac{f^{\prime}(s)}{s}\left(s-\ell_{c}\right) d s \\
& =\frac{c}{b}\left(f\left(\ell_{0}\right)-f\left(\ell_{c}\right)\right)-\frac{c}{b} \ell_{c} \int_{\ell_{c}}^{\ell_{0}} \frac{1}{s} \rho(s) d s \\
& \geq \frac{c}{b}\left(\lambda-2 \ell_{c} \int_{0}^{\infty} \frac{1}{r} \rho(r) d r\right)
\end{aligned}
$$

which proves the lemma.

We apply this lemma to (3.22), with $\rho$ being the probability distribution of $\left|x_{1}-x_{2}\right|$ in the state $\Psi$. We conclude that, if $\Psi$ satisfies (3.21), then

$$
\left\langle\Psi\left|\frac{1}{\left|x_{1}-x_{2}\right|}\right| \Psi\right\rangle \geq \frac{\lambda(U-2 \alpha(1+\epsilon))}{\frac{\pi^{2}}{2\left(1-b^{-1}\right)^{2}}+2 \ell_{c}(U-2 \alpha(1+\epsilon))} .
$$

This implies (3.2).

\section{Many Polarons}

We recall that the Hamiltonian $H_{U}^{(N)}$ for $N$ particles is given by (3.1) and that its ground state and minimum break-up energy were defined in (1.5) and (1.6). The analogue of Theorem 2 in the $N$-particle case is as follows.

Theorem 3 (Upper bound on the $N$-polaron radius). For any $N \geq 2$ and $\epsilon>0$ there is a constant $C_{\epsilon}(N)>0$ such that for all $0<2 \alpha(1+\epsilon)<U$ with $E_{U}^{(N)}(\alpha)<\widetilde{E}_{U}^{(N)}(\alpha)$ and all states $\Psi$

$$
\left\langle\Psi\left|\frac{1}{\max _{i \neq j}\left|x_{i}-x_{j}\right|}\right| \Psi\right\rangle \geq \frac{U-2 \alpha(1+\epsilon)}{C_{\epsilon}(N)(1+U / \alpha)} \frac{\left\langle\Psi\left|\widetilde{E}_{U}^{(N)}(\alpha)-H_{U}^{(N)}\right| \Psi\right\rangle}{\widetilde{E}_{U}^{(N)}(\alpha)-E_{U}^{(N)}(\alpha)} .
$$

Since (4.1) holds for all $\Psi$, it can be reformulated as an operator inequality. The bound is non-trivial only for states $\Psi$ with $\left\langle\Psi\left|H_{U}^{(N)}\right| \Psi\right\rangle<\widetilde{E}_{U}^{(N)}(\alpha)$, however, which exist since $E_{U}^{(N)}(\alpha)<\widetilde{E}_{U}^{(N)}(\alpha)$ by assumption. For approximate ground states, satisfying

$$
\left\langle\Psi\left|H_{U}^{(N)}\right| \Psi\right\rangle \leq(1-\lambda) \widetilde{E}_{U}^{(N)}(\alpha)+\lambda E_{U}^{(N)}(\alpha)
$$

for some $\lambda>0$, (4.1) gives a uniform upper bound on the radius of the multipolaron system. The bound depends only on the value of $\lambda$ and does not explode as $U \rightarrow U_{c}$. 
Proof. We perform a localization similar to that in the two-polaron case, but with $\left|x_{1}-x_{2}\right|$ replaced by the maximum of $\left|x_{i}-x_{j}\right|$ over all particle pairs $(i, j)$. Let $\varphi_{i}$ be given as in (3.4)-(3.5), for some $\ell>0$ and $b>1$. We shall apply (3.6) with

$$
t=\max _{i \neq j}\left|x_{i}-x_{j}\right| .
$$

By the IMS localization formula,

$$
\begin{aligned}
\left\langle\Psi\left|H_{U}^{(N)}\right| \Psi\right\rangle & =\sum_{j \geq 0}\left\langle\left.\Psi \varphi_{j}(t)\left|H_{U}^{(N)}-\sum_{i=1}^{N} \sum_{k \geq 0}\right| \nabla_{i} \varphi_{k}(t)\right|^{2} \mid \Psi \varphi_{j}(t)\right\rangle \\
& =: \sum_{j \geq 0} e_{j}\left\|\Psi \varphi_{j}(t)\right\|^{2} .
\end{aligned}
$$

Moreover, for almost every $X \in \mathbb{R}^{3 N}$,

$$
\sum_{i=1}^{N} \sum_{k \geq 0}\left|\nabla_{i} \varphi_{k}(t)\right|^{2}=2 \sum_{k \geq 0}\left|\varphi_{k}^{\prime}(t)\right|^{2},
$$

which can be bounded as in (3.8) on the support of $\varphi_{j}$. In particular,

$$
e_{0} \geq E_{U}^{(N)}(\alpha)-\frac{\pi^{2}}{2(\ell-\ell / b)^{2}} .
$$

For $j \geq 1$, we now proceed with the one-particle localization as in the twopolaron case, localizing particle $i$ in a ball of radius $b^{j} L$ centered at $u_{i}$, for suitably chosen $L>0$. More precisely, with $\chi$ given in (3.10), let

$$
\Psi_{j, \mathbf{u}}(X)=\Psi(X) \varphi_{j}(t)\left(b^{j} L\right)^{-3 N / 2} \prod_{i=1}^{N} \chi\left(b^{-j}\left(x_{i}-u_{i}\right) / L\right)
$$

where we denote $\mathbf{u}=\left(u_{1}, \ldots, u_{N}\right)$. We have

$$
\left\|\Psi \varphi_{j}(t)\right\|^{2}=\int_{\mathbb{R}^{3 N}} d \mathbf{u}\left\|\Psi_{j, \mathbf{u}}\right\|^{2}
$$

and, again using the IMS formula,

$$
\left\langle\Psi \varphi_{j}(t)\left|H_{U}^{(N)}\right| \Psi \varphi_{j}(t)\right\rangle=\int_{\mathbb{R}^{3 N}} d \mathbf{u}\left\langle\Psi_{j, \mathbf{u}}\left|H_{U}^{(N)}-\frac{N \pi^{2}}{b^{2 j} L^{2}}\right| \Psi_{j, \mathbf{u}}\right\rangle .
$$

All particles are now supported in balls $B_{i}$ of radius $b^{j} L$ centered at $u_{i}$. Moreover, $\Psi_{j, \mathbf{u}}$ is nonzero only if $\max _{i \neq k}\left|u_{i}-u_{k}\right| \geq b^{j-2} \ell-2 b^{j} L$. In particular, if we draw open balls of radius $R$ around all the $u_{i}$, the resulting set is not connected as long as $(N-1) 2 R \leq b^{j-2} \ell-2 b^{j} L$. Hence it is possible to split the particles into two clusters, in such a way that the distance between any two balls in different clusters is at least as big as

$$
\frac{1}{N-1}\left(b^{j-2} \ell-2 b^{j} L\right)-2 b^{j} L=\frac{1}{N-1}\left(b^{j-2} \ell-2 N b^{j} L\right) .
$$

We shall choose $L<\ell /\left(2 N b^{2}\right)$ to make this positive.

The analogue of Lemma 3 that we need now is the following. 
Lemma 5. Assume that $\Psi$ is normalized and supported in the set $B_{1} \times \cdots \times B_{N}$, where the $B_{i}$ are balls of radius $R$. Assume that, for some $1 \leq n \leq N-1$, the distances $d_{i k}$ between balls $B_{i}$ for $i \leq n$ and $B_{k}$ for $k \geq n+1$ are positive. Then, for all $\epsilon>0$,

$$
\begin{aligned}
\left\langle\Psi\left|H_{U}^{(N)}\right| \Psi\right\rangle \geq & E_{U}^{(n)}(\alpha)+E_{U}^{(N-n)}(\alpha) \\
& +\sum_{i=1}^{n} \sum_{k=n+1}^{N}\left(\left\langle\Psi\left|\frac{U-2 \alpha(1+\epsilon)}{\left|x_{i}-x_{k}\right|}\right| \Psi\right\rangle-\frac{2 \alpha}{d_{i k}}+\frac{2 \alpha(1+\epsilon)}{d_{i k}+4 R}\right) .
\end{aligned}
$$

Using the path integral representation of the ground state energy, the proof is easy; we refer to $[5$ for details.

We relabel the particles such that particles $1 \leq i \leq n$ belong to one cluster, and $n+1 \leq k \leq N$ to the other. The distances $d_{i k}$ are all bounded from below by (4.9). They are also bounded above by $\ell b^{j}$ on the support of $\Psi_{j}$. With

$$
D_{j}:=\left[(N-1)^{-1}\left(b^{j-2} \ell-2 N b^{j} L\right), \ell b^{j}\right]
$$

we conclude that

$$
\begin{aligned}
e_{j} \geq & \widetilde{E}_{U}^{(N)}(\alpha)+(N-1)\left\|\Psi_{j}\right\|^{-2}\left\langle\Psi_{j}\left|\frac{U-2 \alpha(1+\epsilon)}{\max _{i \neq k}\left|x_{i}-x_{k}\right|}\right| \Psi_{j}\right\rangle \\
& +(N-1) \min _{d \in D_{j}}\left(-\frac{2 \alpha}{d}+\frac{2 \alpha(1+\epsilon)}{d+4 b^{j} L}\right)-b^{-2 j}\left(\frac{b^{2} \pi^{2}}{2(\ell-\ell / b)^{2}}+\frac{N \pi^{2}}{L^{2}}\right) .
\end{aligned}
$$

Here, we have assumed that the minimum over $d$ in the second line is non-negative, in which case we can use that $n(N-n) \geq(N-1)$ for $1 \leq n \leq N-1$. We can now argue as in the two-polaron case that, for suitably chosen $L$, the sum of the terms in the second line is positive for $\ell$ large enough. In fact, with $L=\delta \ell /(N-1)$ and

$$
\kappa_{\epsilon}:=\min _{b^{-2}-2 \delta N /(N-1) \leq d \leq N-1}\left(-\frac{1}{d}+\frac{1+\epsilon}{d+4 \delta}\right)
$$

(which is positive for small enough $\delta$ ) this is the case for all $j \geq 1$ if

$$
\ell \geq \ell_{c}:=\frac{\pi^{2}}{\alpha \kappa_{\epsilon}(N-1)^{2}}\left(\frac{b}{4\left(1-b^{-1}\right)^{2}}+\frac{N}{2 b \delta^{2}}\right) \text {. }
$$

Recall that $t=\max _{i \neq k}\left|x_{i}-x_{k}\right|$. We have thus shown the operator inequality

$$
\begin{aligned}
H_{U}^{(N)}-\widetilde{E}_{U}^{(N)}(\alpha) \geq & \left(E_{U}^{(N)}-\widetilde{E}_{U}^{(N)}(\alpha)-\frac{\pi^{2}}{2(\ell-\ell / b)^{2}}\right) \theta(\ell-t) \\
& +(N-1) \frac{U-2 \alpha(1+\epsilon)}{t} \theta(t-\ell)
\end{aligned}
$$

for all $\ell \geq \ell_{c}$. The remainder of the proof is as in the two-particle case, applying Lemma 4 to the probability distribution of $t$ in a state $\Psi$.

\section{The Pekar-Tomasevich approximation}

Let us recall, for the reader's convenience, the definition and basic properties of the PT model [5]. There is a non-linear differential-integral variational principle associated with the polaron problem, which gives the exact ground state energy in the limit $\alpha \rightarrow \infty$. This variational problem was investigated in detail by Pekar [17. 
Pekar and Tomasevich (PT) [18] generalized it to the bipolaron, and the extension to $N$-polarons obviously follows from $[18$.

The PT functional is the result of a variational calculation and therefore gives an upper bound to the ground state energy $E_{U}^{(N)}(\alpha)$. In order to compute $\left\langle\Psi, H_{U}^{(N)} \Psi\right\rangle$, one takes a $\Psi$ of the form $\psi \otimes \Phi$ where $\psi \in L^{2}\left(\mathbb{R}^{3 N}\right), \Phi \in \mathcal{F}$, and both $\psi$ and $\Phi$ are normalized. For a given $\psi$ it is easy to compute the optimum $\Phi$, and one ends up with the functional

$$
\begin{aligned}
\mathcal{P}_{U}^{(N)}[\psi]:= & \sum_{i=1}^{N} \int_{\mathbb{R}^{3 N}}\left|\nabla_{i} \psi\right|^{2} d X+U \sum_{i<j} \int_{\mathbb{R}^{3 N}} \frac{|\psi(X)|^{2}}{\left|x_{i}-x_{j}\right|} d X \\
& -\alpha \iint_{\mathbb{R}^{3} \times \mathbb{R}^{3}} \frac{\rho_{\psi}(x) \rho_{\psi}(y)}{|x-y|} d x d y,
\end{aligned}
$$

where $d X=\prod_{k=1}^{N} d x_{k}$, and

$$
\rho_{\psi}(x)=\sum_{i=1}^{N} \int_{\mathbb{R}^{3(N-1)}}\left|\psi\left(x_{1}, \ldots, x, \ldots, x_{N}\right)\right|^{2} d x_{1} \cdots \widehat{d x_{i}} \cdots d x_{N}
$$

with $x$ at the $i$-th position, and $\widehat{d x}_{i}$ meaning that $d x_{i}$ has to be omitted in the product $\prod_{k=1}^{N} d x_{k}$. The ground state energy is

$$
\mathcal{E}_{U}^{(N)}(\alpha)=\inf \left\{\mathcal{P}_{U}^{(N)}[\psi]: \int_{\mathbb{R}^{3 N}}|\psi|^{2} d X=1\right\} .
$$

The variational argument above gives the upper bound

$$
E_{U}^{(N)}(\alpha) \leq \mathcal{E}_{U}^{(N)}(\alpha)=\mathcal{E}_{U / \alpha}^{(N)}(1) \alpha^{2} .
$$

(The equality follows by scaling.) For $N=1$ this upper bound is due to Pekar; numerically, one has $\mathcal{E}^{(1)}(\alpha) \approx-(0.109) \alpha^{2}$ [15. Moreover, the minimization problem for $\mathcal{E}^{(1)}(\alpha)$ has a unique minimizer (up to translations), see [11].

The upper bound for $N=1$ was widely understood to be asymptotically exact for large $\alpha$. A proof of this was finally achieved by Donsker and Varadhan [4, using the functional integral representation and large deviation theory. Later, this fact was rederived in [14 by operator methods, and it was shown that the error was no worse than $\alpha^{9 / 5}$ for large $\alpha$. The fact that for fixed ratio $\nu=U / \alpha \geq 0$ and for $N=2$ one has

$$
\lim _{\alpha \rightarrow \infty} \alpha^{-2} E_{U}^{(N)}(\alpha)=\mathcal{E}_{\nu}^{(N)}(\alpha=1)
$$

was first noted in [16. This is also valid for arbitrary $N$.

We now address the question of whether the infimum $\mathcal{E}_{U}^{(N)}(\alpha)$ is attained, that is, whether there is a minimizer. It was proved in [10] that this is the case provided $\mathcal{E}_{U}^{(N)}(\alpha)<\tilde{\mathcal{E}}_{U}^{(N)}(\alpha)$. This minimum break-up energy is defined as before by

$$
\tilde{\mathcal{E}}_{U}^{(N)}(\alpha)=\min _{1 \leq n \leq N-1}\left(\mathcal{E}_{U}^{(n)}(\alpha)+\mathcal{E}_{U}^{(N-n)}(\alpha)\right) .
$$

Our next theorem gives an answer in the case of equality, i.e., when $\mathcal{E}_{U}^{(N)}(\alpha)=$ $\tilde{\mathcal{E}}_{U}^{(N)}(\alpha)$. 
Theorem 4. Let $U_{c}>0$ be such that $\mathcal{E}_{U_{c}}^{(N)}(\alpha)=\tilde{\mathcal{E}}_{U_{c}}^{(N)}(\alpha)$ and assume that there is a sequence $U_{n} \rightarrow U_{c}$ such that the infimum $\mathcal{E}_{U_{n}}^{(N)}(\alpha)$ is attained. Then the infimum $\mathcal{E}_{U_{c}}^{(N)}(\alpha)$ is attained.

It is important in our proof that any $U_{c}$ as in Theorem 4 satisfies $U_{c}>2 \alpha$, as shown in [10. The key input in our proof is once again a bound on the maximal distance between the particles.

Proposition 2. For any $\nu>1$ and $N \geq 2$ there is an $\ell_{0}<\infty$ and a $\delta_{0}>0$ such that, whenever $U>2 \alpha \nu$ and $\psi_{U}$ is a minimizer for $\mathcal{E}_{U}^{(N)}(\alpha)$, then one has

$$
\int_{\mathbb{R}^{3 N}} \theta\left(\sum_{i, j}\left|x_{i}-x_{j}\right|^{2} \leq \ell_{0}^{2}\right) \psi_{U}^{2} d X \geq \delta_{0}
$$

The proof is similar to that of Theorem 3 and is omitted. We only note that, since we assume that there is a minimizer, we can take $\lambda=1$ in (3.21) and therefore the simple version (2.5) of the calculus lemma suffices and yields (5.6). One also needs to linearize the functional, as we did in [5] and as we will do later in the proof of Theorem 4 .

Proposition 3. Let $U_{n}$ be as in Theorem 4 and let $\psi_{n}$ be the corresponding minimizers. Then there is a non-zero $\psi \in H^{1}\left(\mathbb{R}^{3 N}\right)$ and a sequence $\left\{a_{n}\right\} \subset \mathbb{R}^{3}$ such that a subsequence of $\psi_{n}\left(x_{1}-a_{n}, \ldots, x_{N}-a_{n}\right)$ converges weakly in $H^{1}\left(\mathbb{R}^{3 N}\right)$ to $\psi$.

Proof. Let $\eta:[0, \infty) \rightarrow[0,1]$ be a smooth, compactly supported function with $\eta(t)=1$ for $t$ in a neighborhood of zero. We shall prove the following: There is an $R>0$, a non-zero $u \in H^{1}\left(\mathbb{R}^{3 N}\right)$ and a sequence $\left\{a_{n}\right\} \subset \mathbb{R}^{3}$ such that a subsequence of $u_{n}\left(x_{1}, \ldots, x_{N}\right)=\eta\left(\sum_{i, j}\left|x_{i}-x_{j}\right|^{2} / R^{2}\right) \psi_{n}\left(x_{1}-a_{n}, \ldots, x_{N}-a_{n}\right)$ converges weakly in $H^{1}\left(\mathbb{R}^{3 N}\right)$ to $u$. From this, one easily derives the statement of the lemma.

We first note that $\left\|u_{n}\right\| \leq\left\|\psi_{n}\right\|=1$ and $\sum_{i}\left\|\nabla_{i} u_{n}\right\|^{2} \leq \sum_{i}\left\|\nabla_{i} \psi_{n}\right\|^{2}+C R^{-2}$ for some $C$ independent of $n$. Hence the sequence $u_{n}$ is bounded in $H^{1}\left(\mathbb{R}^{3 N}\right)$, and the assertion will follow by an easy extension of [12, Thm. 8.10], provided we can show that $u_{n}$ does not converge to zero in measure.

To prove this, we use the Euler-Lagrange equation satisfied by the functions $\psi_{n}$,

$$
\left(\sum_{n=1}^{N}\left(p_{i}^{2}-\sqrt{\alpha} \phi_{n}\left(x_{i}\right)\right)+U \sum_{i<j} \frac{1}{\left|x_{i}-x_{j}\right|}\right) \psi_{n}=\lambda_{n} \psi_{n},
$$

with $\phi_{n}=2 \sqrt{\alpha} \rho_{\psi_{n}} *|x|^{-1}$ and $\lambda_{n}=\mathcal{E}_{U_{n}}^{(N)}(\alpha)-\alpha \iint \rho_{\psi_{n}}(x)|x-y|^{-1} \rho_{\psi_{n}}(y) d x d y$. Multiplying the equation by $\eta\left(\sum_{i, j}\left|x_{i}-x_{j}\right|^{2} / R^{2}\right)^{2} \psi_{n}\left(x_{1}, \ldots, x_{N}\right)$ and integrating we find that

$$
\begin{aligned}
& \sum_{i=1}^{N} \int_{\mathbb{R}^{3 N}}\left(\left|\nabla_{i} u_{n}\right|^{2}-\sqrt{\alpha} \phi_{n}\left(x_{i}\right) u_{n}^{2}\right) d X+U \sum_{i<j} \int_{\mathbb{R}^{3 N}} \frac{u_{n}^{2}}{\left|x_{i}-x_{j}\right|} d X \\
& \quad \leq\left(\lambda_{n}+C R^{-2}\right) \int_{\mathbb{R}^{3 N}} u_{n}^{2} d X .
\end{aligned}
$$

Since $\liminf _{n \rightarrow \infty} \lambda_{n} \leq \mathcal{E}_{U_{c}}^{(N)}(\alpha)<0$ we can choose $R>0$ sufficiently large such that $\liminf _{n \rightarrow \infty}\left(\lambda_{n}+C R^{-2}\right)<0$. Moreover, by our key estimate (5.6) we know that, after increasing $R$ if necessary, we have

$$
\liminf _{n \rightarrow \infty} \int_{\mathbb{R}^{3 N}} u_{n}^{2} d X>0 .
$$


(In order to apply Proposition 2 we use the fact that $U_{c}>2 \alpha$, see [10.) From this we conclude that the only negative term on the left side of (5.7) cannot vanish in the limit, i.e.,

$$
\liminf _{n \rightarrow \infty} \sum_{i=1}^{N} \int_{\mathbb{R}^{3 N}} \phi_{n}\left(x_{i}\right) u_{n}^{2} d X>0 .
$$

We now use the 'pqr theorem' [12, Ex. 2.22] to conclude from (5.8) that $\left\{u_{n}\right\}$ does not converge to zero in measure. This theorem states, quite generally, that for any $1 \leq p<q<r \leq \infty$ and for any constant $C<\infty$ there are constants $\epsilon, \delta>0$ such that if a function $f$ satisfies $\|f\|_{p} \leq C,\|f\|_{r} \leq C$ and $\|f\|_{q} \geq C^{-1}$, then $|\{|f| \geq \epsilon\}|>\delta$.

Returning to our concrete situation, we note that, since $\left\{u_{n}\right\}$ is uniformly bounded in $H^{1}\left(\mathbb{R}^{3 N}\right)$, we known from Sobolev inequalities that it is so in $L^{p}\left(\mathbb{R}^{3 N}\right)$ as well for any $2 \leq p \leq 6 N /(3 N-2)$. To apply the 'pqr theorem' we need to show that $\left\|u_{n}\right\|_{p}$ is uniformly bounded away from zero for some $2<p<6 N /(3 N-2)$. First, note that, since $\left(\sqrt{\rho_{n}}\right)$ is uniformly bounded in $H^{1}\left(\mathbb{R}^{3}\right), \phi_{n}$ is uniformly bounded in $L^{q}\left(\mathbb{R}^{3}\right)$ for any $3<q \leq \infty$. Now we choose $C>0$ such that $\eta(t)=0$ for $t \geq C$ and we put

$$
g_{n}(X)=\theta\left(C R^{2}-\sum_{i, j}\left|x_{i}-x_{j}\right|^{2}\right) \sum_{i} \phi_{n}\left(x_{i}\right) .
$$

The sequence $\left\{g_{n}\right\}$ is uniformly bounded in $L^{q}\left(\mathbb{R}^{3 N}\right)$ for any $3<q \leq \infty$, and by (5.8) we have

$$
0<\delta \leq \sum_{i=1}^{N} \int_{\mathbb{R}^{3 N}} \phi_{n}\left(x_{i}\right) u_{n}^{2} d X=\int g_{n} u_{n}^{2} d X \leq\left\|g_{n}\right\|_{q}\left\|u_{n}^{2}\right\|_{q^{\prime}}
$$

where $q^{-1}+q^{-1}=1$. This shows that $\left(u_{n}\right)$ is uniformly bounded away from zero in $L^{2 q^{\prime}}\left(\mathbb{R}^{3 N}\right)$, and we can choose $3<q<\infty$ in such a way that $2<2 q^{\prime}<6 N /(3 N-2)$. Hence the 'pqr theorem' implies that $u_{n}$ does not tend to zero in measure, which completes the proof of the lemma.

We now turn to the proof of Theorem 4 An important ingredient, which we have already used in [5], is that the energy functional can be linearized. In order to state this precisely we define for any pair $(\psi, \phi) \in H^{1}\left(\mathbb{R}^{3 N}\right) \cap \dot{H}^{1}\left(\mathbb{R}^{3}\right)$ (with $\psi$ not necessarily normalized) the energy functional

$$
\begin{aligned}
\mathcal{P}_{U}[\psi, \phi]:= & \sum_{i=1}^{N} \int_{\mathbb{R}^{3 N}}\left(\left|\nabla_{i} \psi\right|^{2}-\sqrt{\alpha} \phi\left(x_{i}\right)|\psi|^{2}\right) d X+U \sum_{i<j} \int_{\mathbb{R}^{3 N}} \frac{|\psi(X)|^{2}}{\left|x_{i}-x_{j}\right|} d X \\
& +\frac{1}{16 \pi} \int_{\mathbb{R}^{3}}|\nabla \phi|^{2} d x \int_{\mathbb{R}^{3 N}}|\psi(X)|^{2} d X
\end{aligned}
$$

The crucial observation is that for any $\psi$ and $\phi$ one has

$$
\mathcal{P}_{U}[\psi, \phi] \geq\|\psi\|^{2} \mathcal{P}_{U}^{(N)}\left[\|\psi\|^{-1} \psi\right],
$$

with equality if and only if $\phi=2 \sqrt{\alpha}|x|^{-1} * \rho_{\psi /\|\psi\|}$. We are now ready to give the

Proof of Theorem 4. Because of Proposition 3, after passing to a subsequence and a translation we may assume that $\left\{\psi_{n}\right\}$ converges weakly in $H^{1}\left(\mathbb{R}^{3 N}\right)$ to $\psi \not \equiv 0$. 
Denoting $\tilde{\psi}_{n}=\psi_{n}-\psi$ and $\phi_{n}=2 \sqrt{\alpha}|x|^{-1} * \rho_{\psi_{n}}$ we claim that

$$
\mathcal{P}_{U_{n}}\left[\psi_{n}, \phi_{n}\right]=\mathcal{P}_{U_{c}}\left[\psi, \phi_{n}\right]+\mathcal{P}_{U_{c}}\left[\tilde{\psi}_{n}, \phi_{n}\right]+o(1) .
$$

Assuming (5.11) for the moment, we now finish the proof of Theorem 4 Indeed, in view of (5.10), we conclude from (5.11) that

$$
\mathcal{E}_{U_{c}}^{(N)}(\alpha)+o(1) \geq\|\psi\|^{2} \mathcal{P}_{U_{c}}^{(N)}\left[\|\psi\|^{-1} \psi\right]+\left\|\tilde{\psi}_{n}\right\|^{2} \mathcal{P}_{U_{c}}^{(N)}\left[\left\|\tilde{\psi}_{n}\right\|^{-1} \tilde{\psi}_{n}\right]+o(1) .
$$

Since $\mathcal{P}_{U_{c}}^{(N)}\left[\left\|\tilde{\psi}_{n}\right\|^{-1} \tilde{\psi}_{n}\right] \geq \mathcal{E}_{U_{c}}^{(N)}(\alpha)$ and since $\left\|\tilde{\psi}_{n}\right\|^{2}=1-\|\psi\|^{2}+o(1)$, we learn that

$$
\mathcal{E}_{U_{c}}^{(N)}(\alpha)+o(1) \geq \mathcal{P}_{U_{c}}^{(N)}\left[\|\psi\|^{-1} \psi\right]+o(1),
$$

that is, $\|\psi\|^{-1} \psi$ is an optimizer at $U=U_{c}$.

It remains to prove (5.11). The analogous assertion separately for the terms $\sum_{i=1}^{N} \int\left|\nabla_{i} \psi\right|^{2} d X$ and $\sum_{i<j} \int \frac{|\psi(X)|^{2}}{\left|x_{i}-x_{j}\right|} d X$ is an easy consequence of weak convergence. Moreover,

$$
\int_{\mathbb{R}^{3}}\left|\nabla \phi_{n}\right|^{2} d x=\int_{\mathbb{R}^{3}}\left|\nabla \phi_{n}\right|^{2} d x \int_{\mathbb{R}^{3 N}}|\psi(X)|^{2}+\int_{\mathbb{R}^{3}}\left|\nabla \phi_{n}\right|^{2} d x \int_{\mathbb{R}^{3 N}}\left|\tilde{\psi}_{n}(X)\right|^{2}+o(1)
$$

by the weak convergence of $\psi_{n}$ and the fact that $\int\left|\nabla \phi_{n}\right|^{2} d x$ is uniformly bounded. Thus it remains to prove that

$$
\int_{\mathbb{R}^{3 N}} \phi_{n}\left(x_{i}\right) \tilde{\psi}_{n} \psi d X=o(1)
$$

for each $i$. To prove this, we let $R>0$ and split the integral on the left side according to whether $|X|_{\infty}=\max \left\{\left|x_{1}\right|, \ldots,\left|x_{N}\right|\right\}$ is bigger or smaller than $R$. In this way we find that

$$
\begin{aligned}
& \left|\int_{\mathbb{R}^{3 N}} \phi_{n}\left(x_{i}\right) \tilde{\psi}_{n} \psi d X\right| \\
& \leq \sup _{k}\left\|\phi_{k}\right\|_{\infty}\left(\sup _{k}\left\|\tilde{\psi}_{k}\right\|\left\|\psi \theta\left(|X|_{\infty} \geq R\right)\right\|+\left\|\tilde{\psi}_{n} \theta\left(|X|_{\infty} \leq R\right)\right\|\right) .
\end{aligned}
$$

Here we also used that $\|\psi\| \leq 1$. The fact that $\left\{\phi_{n}\right\}$ is uniformly bounded in $L^{\infty}\left(\mathbb{R}^{3}\right)$ follows from the fact that $\left\{\sqrt{\rho_{\psi_{n}}}\right\}$ is uniformly bounded in $H^{1}\left(\mathbb{R}^{3}\right)$. We can make the first term on the right side of (5.13) as small as we like by choosing $R$ large. On the other hand, for any fixed $R$, the sequence $\left\{\tilde{\psi}_{n} \theta\left(|X|_{\infty} \leq R\right)\right\}$ tends to zero in $L^{2}\left(\mathbb{R}^{3 N}\right)$ by the Rellich-Kondrashov Theorem [12, Thm. 8.9], and therefore we can make the second term on the right side of (5.13) as small as we like by choosing $n$ large. This completes the proof of (5.12) and therefore of Theorem 4.

Acknowledgments. We are grateful to Herbert Spohn for making us aware of this problem. Partial financial support from the U.S. National Science Foundation through grant PHY-0965859 (E.L.) and the NSERC (R.S.) is gratefully acknowledged. 


\section{REFERENCES}

[1] R. D. Benguria, G. A. Bley, Exact asymptotic behavior of the Pekar-Tomasevich functional, J. Math. Phys. 52 (2011), 052110.

[2] R. D. Benguria, R. L. Frank, E. H. Lieb, in preparation.

[3] H. A. Bethe, Berechnung der Elektronenaffinität des Wasserstoffs, Z. Physik 57 (1929), 815.

[4] M. Donsker, S. R. S. Varadhan, Asymptotics for the polaron. Comm. Pure Appl. Math. 36 (1983), 505-528.

[5] R. L. Frank, E. H. Lieb, R. Seiringer, L. E. Thomas, Bi-polaron and N-polaron binding energies. Phys. Rev. Lett. 104, 210402 (2010). Stability and Absence of Binding for MultiPolaron Systems, Publ. Math. IHES 113 (2011), no. 1, 39-67.

[6] H. Fröhlich, Theory of electrical breakdown in ionic crystals. Proc. R. Soc. Lond. A 160 (1937), 230-241.

[7] B. Gerlach, H. Löwen, Absence of phonon-induced localization for the free optical polaron and the corresponding Wannier exciton-phonon system. Phys. Rev. B 37 (1988), 8042-8047.

[8] M. Griesemer, J. S. Møller, Bounds on the minimal energy of translation invariant $N$-polaron systems. Comm. Math. Phys. 297, 283-297 (2010).

[9] M. Hoffmann-Ostenhof, T. Hoffmann-Ostenhof, B. Simon, A multiparticle Coulomb system with bound state at threshold, J. Phys. A 16, 1125-1131 (1983).

[10] M. Lewin, Geometric methods for nonlinear many-body quantum systems, J. Funct. Anal. 260, 3535-3595 (2011).

[11] E. H. Lieb, Existence and uniqueness of the minimizing solution of Choquard's nonlinear equation. Studies in Appl. Math. 57 (1976/77), no. 2, 93-105.

[12] E. H. Lieb, M. Loss, Analysis, volume 14 of Graduate Studies in Mathematics. Amer. Math. Soc., Providence, RI, second edition, 2001.

[13] E. H. Lieb, R. Seiringer, The stability of matter in quantum mechanics. Cambridge (2010).

[14] E. H. Lieb, L. E. Thomas, Exact ground state energy of the strong-coupling polaron. Comm. Math. Phys. 183, no. 3, 511-519 (1997). Erratum: ibid. 188, no. 2, 499-500 (1997).

[15] S. J. Miyake, Strong coupling limit of the polaron ground state. J. Phys. Soc. Jpn. 38 (1975), 181-182.

[16] T. Miyao, H. Spohn, The bipolaron in the strong coupling limit. Ann. Henri Poincaré 8 (2007), $1333-1370$.

[17] S. I. Pekar, Research in electron theory of crystals. United States Atomic Energy Commission, Washington, DC, 1963.

[18] S. I. Pekar, O. F. Tomasevich, Theory of F centers. Zh. Eksp. Teor. Fys. 21 (1951), 12181222 .

[19] M. A. Smondyrev, V.M. Fomin, Pekar-Fröhlich bipolarons. In: Polarons and applications, Proceedings in Nonlinear Science, V.D. Lakhno, ed., Wiley (1994).

[20] M. A. Smondyrev, G. Verbist, F. M. Peeters, J. T. Devreese, Stability of multi polaron matter, Phys. Rev. B 47 (1993), 2596-2601.

[21] S. G. Suprun, B. Ya. Moizhes, Fiz. Tverd. Tela 24 (1982), 1571; Sov. Physc. Semicond. 16 (1982), 700.

[22] G. Verbist, F. M. Peeters, J. T. Devreese, Large bipolarons in two and three dimensions. Phys. Rev. B 43 (1991), 2712-2720.

[23] G. Verbist, M. A. Smondyrev, F. M. Peeters, J. T. Devreese, Strong-coupling analysis of large bipolarons in two and three dimensions. Phys. Rev. B 45 (1992), 5262-5269.

[24] G. M. Zhislin, A study of the spectrum of the Schrödinger operator for a system of several particles. (Russian) Trudy Moskov. Mat. Obsc. 9 (1960) 81-120. 
R.L. Frank, Department of Mathematics, Princeton University, Washington Road, PRINCETON, NJ 08544, USA

E-mail address: rlfrank@math.princeton.edu

E.H. Lieb, Departments of Mathematics and Physics, Princeton University, P.O. Box 708, Princeton, NJ 08544, USA

E-mail address: lieb@princeton.edu

R. Seiringer, Department of Mathematics and Statistics, McGill University, 805 Sherbrooke Street West, Montreal, QC H3A 2K6, Canada

E-mail address: rseiring@math.mcgill.ca 